Acta Crystallographica Section E

Structure Reports

Online

ISSN 1600-5368

\section{Mepazinium picrate}

H. S. Yathirajan, ${ }^{a}$ M. A. Ashok, ${ }^{a}$

B. Narayana Achar and Michael Bolte $^{b_{*}}$

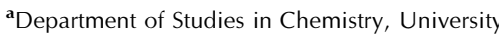
of Mysore, Manasagangotri, Mysore 570 006, India, and ${ }^{\mathbf{b}}$ Institut für Anorganische Chemie, J. W. Goethe-Universität Frankfurt, Max-vonLaue-Strasse 7, 60438 Frankfurt/Main, Germany

Correspondence e-mail:

bolte@chemie.uni-frankfurt.de

\section{Key indicators}

Single-crystal X-ray study

$T=173 \mathrm{~K}$

Mean $\sigma(\mathrm{C}-\mathrm{C})=0.002 \AA$

$R$ factor $=0.036$

$w R$ factor $=0.097$

Data-to-parameter ratio $=13.4$

For details of how these key indicators were automatically derived from the article, see http://journals.iucr.org/e.
The title compound [systematic name: 1-methyl-3-(10Hphenothiazin-10-ylmethyl)piperidinium 2,4,6-trinitrophenolate], $\mathrm{C}_{19} \mathrm{H}_{23} \mathrm{~N}_{2} \mathrm{~S}^{+} \mathrm{C}_{6} \mathrm{H}_{2} \mathrm{~N}_{3} \mathrm{O}_{7}{ }^{-}$, is a pharmacologically active compound. The dihedral angle between the two outer aromatic rings of the phenothiazine unit is $41.58(7)^{\circ}$. The crystal packing is stabilized by $\mathrm{N}-\mathrm{H} \cdots \mathrm{O}$ hydrogen bonds and several weak $\mathrm{C}-\mathrm{H} \cdots \mathrm{O}$ contacts.

\section{Comment}

Mepazine, chemically 10-[(1-methyl-3-piperidyl)methyl]-10Hphenothiazine, is an antipsychotic drug (Tedeschi et al., 1958). In continuation of our work on the crystallization of phenothiazine drugs with picrate (Yathirajan et al., 2007), we present here the formation of a salt by the interaction between 10-[(1-methyl-3-piperidyl)methyl]-10H-phenothiazine hydrochloride and 2,4,6-trinitrophenol in an aqueous medium.<smiles>C[NH+]1CCCC(CN2c3ccccc3Sc3ccccc32)C1</smiles>

(I)

A perspective view of the structure of (I) is shown in Fig. 1. Bond lengths and angles can be regarded as normal (Cambridge Structural Database, Version 5.28, November 2006; Allen, 2002; Mogul, Version 1.1; Bruno et al., 2004). The

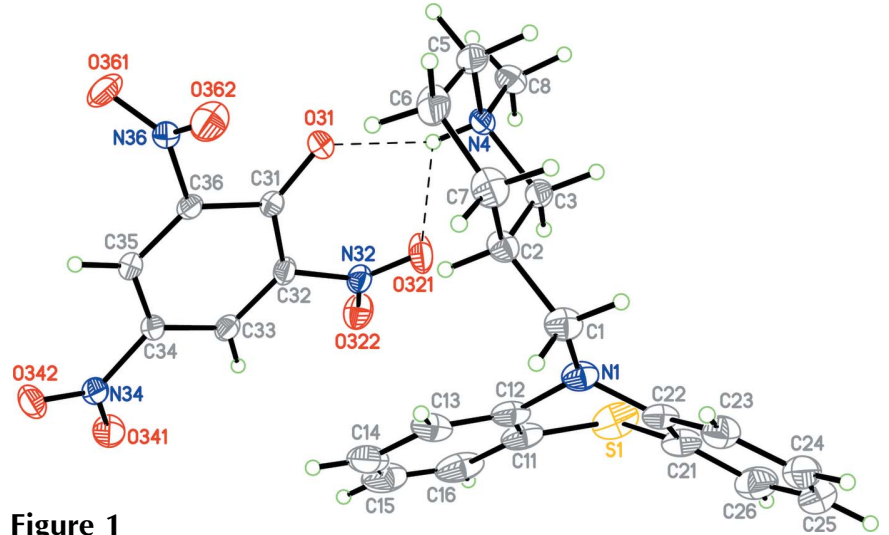

Figure 1

The molecular structure of the title compound with the atom numbering. Displacement ellipsoids are drawn at the $30 \%$ probability level. Dashed lines indicate hydrogen bonds.
Received 23 February 2007 Accepted 1 March 2007
(C) 2007 International Union of Crystallography All rights reserved 
dihedral angle between the two aromatic rings of the phenothiazine unit is $41.58(7)^{\circ}$. The piperidyl ring adopts a chair conformation with both substituents in equatorial positions. The crystal packing is stabilized by $\mathrm{N}-\mathrm{H} \cdots \mathrm{O}$ hydrogen bonds and several weak $\mathrm{C}-\mathrm{H} \cdots \mathrm{O}$ contacts (Table 1 ).

\section{Experimental}

Mepazine hydrochloride monohydrate $(0.7300 \mathrm{~g}, 0.02 \mathrm{M})$ and picric acid $(0.4606 \mathrm{~g}, 0.02 \mathrm{M})$ were dissolved separately in doubly distilled water $(100 \mathrm{ml})$. The solutions were mixed and stirred in a beaker. A bright-yellow salt was formed instantaneously at room temperature. The separated salt was filtered off, washed thoroughly with doubly distilled water and dried in a vacuum desiccator over phosphorus pentoxide. The compound was recrystallized from ethanol (m.p. $379 \mathrm{~K})$.

\section{Crystal data}

$\begin{array}{ll}\mathrm{C}_{19} \mathrm{H}_{23} \mathrm{~N}_{2} \mathrm{~S}^{+} \cdot \mathrm{C}_{6} \mathrm{H}_{2} \mathrm{~N}_{3} \mathrm{O}_{7}{ }^{-} & \gamma=102.419(7)^{\circ} \\ M_{r}=539.56 & V=1251.8(2) \AA^{3} \\ \text { Triclinic, } P \overline{1} & Z=2 \\ a=8.3584(9) \AA & \text { Mo } K \alpha \text { radiation } \\ b=10.7782(9) \AA & \mu=0.19 \mathrm{~mm}^{-1} \\ c=14.5062(13) \AA & T=173(2) \mathrm{K} \\ \alpha=93.524(7)^{\circ} & 0.45 \times 0.45 \times 0.40 \mathrm{~mm}\end{array}$

\section{Data collection}

Stoe IPDSII two-circle diffractometer

Absorption correction: multi-scan (MULABS; Spek, 2003; Blessing, 1995) $T_{\min }=0.911, T_{\max }=0.920$

\section{Refinement}

$R\left[F^{2}>2 \sigma\left(F^{2}\right)\right]=0.037$

$w R\left(F^{2}\right)=0.098$

$S=1.03$

4656 reflections

348 parameters

12751 measured reflections 4656 independent reflections 3824 reflections with $I>2 \sigma(I)$ $R_{\text {int }}=0.037$ independent and constrained refinement

$\Delta \rho_{\max }=0.24{\mathrm{e} \AA^{-3}}^{-3}$
Table 1

Hydrogen-bond geometry $\left(\AA,^{\circ}\right)$.

\begin{tabular}{|c|c|c|c|c|}
\hline$D-\mathrm{H} \cdots A$ & $D-\mathrm{H}$ & $\mathrm{H} \cdots A$ & $D \cdots A$ & $D-\mathrm{H} \cdots A$ \\
\hline $\mathrm{N} 4-\mathrm{H} 4 \cdots \mathrm{O} 31$ & $0.89(2)$ & $1.84(2)$ & $2.6879(17)$ & $158.2(19)$ \\
\hline $\mathrm{N} 4-\mathrm{H} 4 \cdots \mathrm{O} 321$ & $0.89(2)$ & $2.46(2)$ & 3.0191 (17) & $121.8(16)$ \\
\hline $\mathrm{C} 3-\mathrm{H} 3 B \cdots \mathrm{O} 321$ & 0.99 & 2.50 & $3.0790(19)$ & 117 \\
\hline $\mathrm{C} 5-\mathrm{H} 5 B \cdots \mathrm{O} 342^{\mathrm{i}}$ & 0.99 & 2.50 & $3.409(2)$ & 152 \\
\hline $\mathrm{C} 6-\mathrm{H} 6 A \cdots \mathrm{O} 362$ & 0.99 & 2.47 & $3.419(2)$ & 160 \\
\hline $\mathrm{C} 6-\mathrm{H} 6 B \cdots \mathrm{O} 341^{\mathrm{ii}}$ & 0.99 & 2.56 & $3.4229(19)$ & 146 \\
\hline $\mathrm{C} 35-\mathrm{H} 35 \cdots \mathrm{O} 322^{\mathrm{iii}}$ & 0.95 & 2.52 & 3.4568 (19) & 168 \\
\hline
\end{tabular}

Symmetry codes: (i) $x-1, y-1, z$; (ii) $x, y-1, z$; (iii) $x+1, y, z$.

$\mathrm{H}$ atoms were found in a difference map. The $\mathrm{H}$ atom bonded to nitrogen was refined freely and all other $\mathrm{H}$ atoms were refined using a riding model, with $\mathrm{C}-\mathrm{H}=0.95-0.99 \AA$ and $U_{\text {iso }}(\mathrm{H})=1.2 U_{\text {eq }}(\mathrm{C})$ or $1.5 U_{\text {eq }}$ (methyl C).

Data collection: $X$-AREA (Stoe \& Cie, 2001); cell refinement: $X$-AREA; data reduction: $X$-AREA; program(s) used to solve structure: SHELXS97 (Sheldrick, 1997); program(s) used to refine structure: SHELXL97 (Sheldrick, 1997); molecular graphics: XP in SHELXTL-Plus (Sheldrick, 1991); software used to prepare material for publication: SHELXL97 and PLATON (Spek, 2003).

MAA thanks the University of Mysore for research facilities.

\section{References}

Allen, F. H. (2002). Acta Cryst. B58, 380-388.

Blessing, R. H. (1995). Acta Cryst. A51, 33-38.

Bruno, I. J., Cole, J. C., Kessler, M., Luo Jie Motherwell, W. D. S., Purkis, L. H., Smith, B. R., Taylor, R., Cooper, R. I., Harris, S. E. \& Orpen, A. G. (2004). J. Chem. Inf. Comput. Sci. 44, 2133-2144.

Sheldrick, G. M. (1991). SHELXTL-Plus. Release 4.1. Siemens Analytical X-ray Instruments Inc., Madison, Wisconsin, USA.

Sheldrick, G. M. (1997). SHELXS97 and SHELXL97. University of Göttingen, Germany.

Spek, A. L. (2003). J. Appl. Cryst. 36, 7-13.

Stoe \& Cie (2001). $X$-AREA. Stoe \& Cie, Darmstadt, Germany.

Tedeschi, D. H., Benigni, J. P., Elder, C. J., Yeager, J. C. \& Flanigan, J. V. (1958). J. Pharmacol. Exp. Ther. 123, 35-38.

$\Delta \rho_{\min }=-0.31{\mathrm{e} \AA^{-3}}^{-3}$
Yathirajan, H. S., Ashok, M. A., Narayana Achar, B. \& Bolte, M. (2007). Acta Cryst. E63, o1432-o1434. 